\title{
Information flow in the mental lexicon during speech planning: evidence from event-related brain potentials
}

\author{
Jörg D. Jescheniak ${ }^{\mathrm{a}, \mathrm{b}, *}$, Anja Hahne ${ }^{\mathrm{a}}$, Herbert Schriefers ${ }^{\mathrm{c}}$ \\ ${ }^{a}$ Max Planck Institute of Cognitive Neuroscience, Stephansstrasse 1A, D-04103 Leipzig, Germany \\ ${ }^{\mathrm{b}}$ University of Potsdam, Potsdam, Germany \\ ${ }^{\mathrm{c}}$ Nijmegen Institute of Cognition and Information, Nijmegen University, Nijmegen, The Netherlands
}

Accepted 11 July 2002

\begin{abstract}
A major issue in speech production research is the question of how speakers retrieve words from the so-called mental lexicon. Current models of lexical retrieval converge on the assumption that category associates of a target word are semantically activated during speech planning. However, the question of whether these competitors are also phonologically activated is less agreed on. Past research has addressed this issue by testing whether lexical retrieval of a picture name (e.g. sheep) affects, or is affected by, the processing of a word that is phonologically related to a semantic category associate to the picture name (e.g. goal, phonologically related to goat). Behavioral studies have failed to obtain such so-called mediated priming effects, but have been questioned on the grounds of possibly insufficient task sensitivity. As such priming effects have reliably been obtained with event-related brain potentials in word recognition, we used this approach for testing these effects in lexical retrieval during speech planning. Our results consistently demonstrate the absence of mediated priming effects, putting strong constraints on the activation flow in the mental lexicon during speech planning.
\end{abstract}

(C) 2002 Elsevier Science B.V. All rights reserved.

Theme: Neural basis of behavior

Topic: Cognition

Keywords: Language; Speech production; Mental lexicon; Lexical access

\section{Introduction}

There is hardly any dispute among researchers that cognitive planning in speaking, when viewed from a global perspective, proceeds in two stages from meaning and syntactic structure to a specification of sound form [6,10,21,22] (but see Ref. [3]). However, as soon as a more detailed perspective is taken, only little consensus remains. Some researchers maintain that semantic and syntactic planning strictly precede phonological planning and that phonological codes are only activated for the words actually to be produced, but not for their semantic competitors [22,23]. This is the core assumption of socalled serial-discrete (or modular) models of lexical access.

\footnotetext{
*Corresponding author. Present address: Max Planck Institute of Cognitive Neuroscience, Stephansstrasse 1A, D-04103 Leipzig, Germany. Tel.: +49-341-9940276; fax: +49-341-9940113.

E-mail address: jeschen@cns.mpg.de (J.D. Jescheniak).
}

Other researchers, by contrast, assume that the activation of semantic-syntactic codes (so-called lemmas) and of phonological codes are more continuous processes, in that phonological activation can begin before semantic-syntactic processing has terminated with the selection of one candidate (forward-cascading models) [25] and may even, via feedback, exert some influence on this selection process at the lemma level (interactive models) [6-9,13].

Evidence in support of the notion that lexical-semantic and syntactic activation is initiated prior to phonological activation comes from behavioral studies using the crossmodal picture-word task, in which participants name pictures of objects while ignoring auditory distractor words. Schriefers et al. [34] found that effects from auditory distractor words either semantically or phonologically related to a to-be-produced picture name followed a different time course, with semantic inhibition effects being obtained only when distractors were presented shortly before picture onset, and phonological facilitation 
effects being obtained only when distractors were presented simultaneously with or slightly after picture onset (although some temporal overlap of the two effects was observed in later picture-word studies $[5,20])$. Subsequent electrophysiological studies using lateralized readiness potentials [32,33,36,37] confirmed the global pattern and provided estimates of the delays with which different lexical codes become available during speech planning. However, these studies do not speak to the issue of whether the sound forms of semantic competitors of a target word are activated during speech planning.

This question has been addressed in a behavioral study by Levelt et al. [23], which used a primed lexical decision task. In this task, participants were asked to perform a lexical decision (word vs. non-word) on an auditory target stimulus while preparing a picture naming response. Lexical decisions were found to be delayed if the auditory target stimulus was the name of a semantic category associate of the prepared picture name (semantic condition, e.g. goat, if the picture showed a sheep) or if it was phonologically related to the prepared picture name (phonological condition, e.g. sheet), when compared to unrelated controls (e.g. house). By contrast, no such effect was obtained if the auditory target stimulus was phonologically related to the name of the semantic category associate of the prepared picture name (mediated condition, e.g. goal). This pattern was taken as support for serial-discrete models, in which only words selected for production become phonologically activated, while lexical competitors, even if they had become semantically activated, remain phonologically inactivated.

In response to this interpretation, it has been voiced repeatedly that the null-effect in the mediated condition might be a consequence of insufficient task sensitivity, which may have missed existing but small effects. Indeed, it has been argued that, on computational grounds, mediated effects must be expected to be substantially smaller than direct (semantic or phonological) effects which were reliably detected with the experimental task. As Dell and O'Seaghdha put it "mediated semantic-phonological effects should be small, but we leave the determination of just how small to future research. The spreading-activation model suggests that there might be a slight effect, whereas the modular two-step account states that there should be none" (Ref. [8], p. 299). By means of computer simulation, Dell and O'Seaghdha $[7,8]$ demonstrated that the results of Levelt et al. [23] need not rule out non-discrete models but can be accommodated to interactive-activation models, given certain constraints on the parameter setting [13]. In line with predictions derived from extensions of such simulations [16], mediated effects were later obtained when testing for the phonological activation of very strong lexical competitors, namely synonyms. Corresponding experiments found that the preparation of a picture naming response (e.g. sofa) primes a subsequently presented target word (e.g. count) that is phonologically related to the picture's synonymous alternative name (e.g. couch)
$[18,25]$. However, the interpretation of this finding remains controversial. It can be seen as evidence that the phonological form of a non-selected lexical competitor always becomes activated, but that such activation is small and only measurable for extreme semantic competition, as is the case between two synonymous names of the same picture [25]. However, it has also been suggested that such a simultaneous activation of multiple phonological codes is confined to situations in which both competitors are appropriate naming responses for a given picture, as is the case for synonyms (e.g. couch-sofa), but not for category associates (e.g. sheep-goat) [19,22]. In such situations, selection of more than one lemma might occur, each of them activating its respective phonological code subsequently. In view of this possibility, it is crucial to firmly establish whether phonological activation of lexical competitors is indeed confined to appropriate name alternatives or can also be obtained for non-appropriate lexical competitors, such as category associates, for advancing the theoretical debate.

In line with the general prediction that mediated effects should be small, O'Seaghdha and Marin [24] reported very subtle mediated semantic-phonological priming effects for category associate competitors when participants performed a speeded lexical decision task on visually presented target stimuli. These effects were statistically confirmed only in a joint analysis across several experiments. As the authors put it, the effect was "near the limit of the naming task" (Ref. [24], p. 226). While these data seem to support the predictions of non-discrete models, it is important to take a closer look at the experimental paradigm used. Unlike Peterson and Savoy (and also Levelt et al.), who used to-be-named pictures as primes and took the response to the target words as an index of the lexical activation resulting from picture name retrieval, O'Seaghdha and Marin used visual words as primes. Therefore, O'Seaghdha and Marin's result may index lexical activation patterns during visual word recognition rather than lexical activation patterns during speech planning.

In summary, the issue of whether mediated priming of semantic category associates occurs during speech planning has not yet been resolved by the above mentioned studies using behavioral measures. In the present study, we addressed this issue by using electrophysiological measures. Such measures have been shown to be sensitive to subtle effects that could not be detected by reaction time measures. In particular, this is the case for two-step mediated priming in visual word recognition, in which the prime word (e.g. lion) and the target word (e.g. stripes) are only related via a mediating word (e.g. tiger) that is not presented in the experimental trial. Chwilla et al. [4] showed that in such priming situations reaction time effects and N400 effects can dissociate. More specifically, using a standard primed lexical decision task, Chwilla et al. obtained mediated priming effects in their electrophysiological measures (a N400 priming effect), but not in 
the simultaneously collected lexical decision reaction times.

Before turning to a description of the experiments proper, a few words on the choice of the experimental task seem to be in place. Thus far, there are only a few ERP studies investigating cognitive aspects of speech planning, and most of these have measured lateralized readiness potentials (LRPs) in binary go-/no-go tasks [32,33,36,37]. This research has shown that the presence and onset of LRPs in different task configurations allows for highresolution time estimates about the availability of different lexical codes during picture name retrieval. However, as the paradigm requires participants to retrieve a particular code such that it can feed a voluntary meta-linguistic decision, it is not easily applied to the issue at hand here, namely the question of whether a certain lexical code (i.e. the phonological code of a semantic category associate) becomes activated automatically during speech planning.

For this reason, we resorted to a different ERP technique developed for tapping such automatic lexical retrieval processes [17]. This technique can be viewed as an 'electrified' version of the behavioral technique used by Levelt et al. [23] and Peterson and Savoy [25]. It combines a delayed picture naming task with a priming procedure. The participants' primary task is to prepare a picture naming response and to execute it as quickly as possible upon receiving a response cue. On the experimental trials, however, an auditory word is presented shortly after the picture and prior to the response cue. In this case, participants are instructed to listen to the word so that they can later decide whether it matches a visual probe word presented at the end of the experimental trial. The auditory word serves as the target to which the brain response is recorded. The prepared naming response is expected to act as a self-generated prime that should exert its influence on the processing of the target word, in particular in the N400 time window (for semantic N400 priming effects in wordword priming studies see Refs. [1,14,15,28]; for phonological N400 priming effects see Refs. [26-30]). In fact, using this paradigm, an attenuated negativity of the ERP waveform to the target word was observed if the prepared picture name and the target word were semantically or phonologically related, starting 400 and $250 \mathrm{~ms}$ after target onset, respectively. Moreover, the phonological effect could be shown to be a specific index of word-form activation during lexical processing; it disappeared if participants performed a non-lexical (natural-size judgment) task on the depicted object [17] ${ }^{1}$.

With this paradigm, we tested for the lexical activation

\footnotetext{
${ }^{1}$ It should be noted that the technique used in the present study, while allowing tapping of automatic activation of different codes during speech planning, is limited in its potential to trace the time course in which these codes become available (as the latter will depend on the point in time at which the relevant phonological or semantic information is delivered by the auditory target word). As the situation is exactly reverse in the case of the LRP approach, the present technique and the LRP technique can best be seen as complementing each other.
}

of semantic category associates during speech planning. Experiment 1 established that semantic and phonological priming effects could reliably be obtained with the selected materials. Experiments 2-4, then, tested for mediated priming effects with these materials. They also included the phonological test conditions to facilitate a comparison across experiments.

\section{Experiment 1}

\subsection{Methods}

\subsubsection{Participants}

A total of 32 healthy participants (14 men and 18 women, with a mean age of 23.5 years, ranging from 19 to 29 years) were tested. In this and all other experiments reported in this article, they were recruited from the participant pool of the Max Planck Institute of Cognitive Neuroscience and had participated in other unrelated ERP experiments previously. All of them were right-handed, native speakers of German, with no known hearing deficit and normal or corrected-to-normal vision. They were paid for their participation (DM 13 per hour, US \$6). Participants were replaced if less than $70 \%$ error- and artifact-free experimental trials were left for the ERP analyses.

\subsubsection{Materials}

Our study included pictures of 48 simple objects which all had one unambiguous name. For each object, three target words were selected. The first one was the name of a close semantic category associate to the object name (e.g. $F u \beta$ [foot], if the picture name was Hand [hand], semantic condition), the second one shared all phonological segments except for the consonantal onset with the object name (e.g. Sand [sand], phonological condition), and the third one shared all phonological segments except for the consonantal onset with the close semantic category associate to the object name (e.g. Mus [pap], mediated semantic-phonological condition) ${ }^{2}$. Unrelated control conditions against which any specific effect of these three related pairs of prime pictures and target words could be evaluated were created by reassigning the pictures to the words. This way, priming effects could be measured on the same target words while additionally ensuring that no incidental differences across prime picture sets contributed to the observed effects. Note that the mediated semanticphonological condition was only used in Experiments 2-4. However, for ease of exposition, the construction of materials for this condition is described here.

\footnotetext{
${ }^{2}$ The non-identical consonantal onset of the target words used in the phonological and the mediated conditions consisted of one phonological segment (as in the example given) or, occasionally, of two phonological segments (e.g. picture name Pferd [horse], with the target words Wert [value], Schwein [pig], and Hain [grove]).
} 
Prime pictures were scaled to fill a square of $\sim 50 \times 50$ $\mathrm{mm}$. Auditory target words were spoken by a female native speaker of German, digitized at a sampling rate of $22 \mathrm{KHz}$, and further processed with sound editing software. The length of these words ranged from 308 to $719 \mathrm{~ms}$, with an average of $543 \mathrm{~ms}$ (S.D. $86 \mathrm{~ms}$ ) (semantic condition: $308-715 \mathrm{~ms}$, with an average of $538 \mathrm{~ms}$ (S.D. $86 \mathrm{~ms}$ ); phonological condition: $324-719 \mathrm{~ms}$, with an average of $538 \mathrm{~ms}$ (S.D. $96 \mathrm{~ms}$ ); mediated condition: 408-712 ms, with an average of $554 \mathrm{~ms}$ (S.D. $76 \mathrm{~ms})$ ). In addition to these experimental materials, a set of nine pictures along with appropriate target words was selected to be used in practice trials.

To maximize the chance of detecting direct and mediated phonological priming effects (phonological and mediated semantic-phonological condition, respectively), the phonologically related and mediated auditory target words were deliberately chosen such that they had maximum phonological overlap with the prime picture name or its close semantic category associate. But in view of this fact, it was necessary to ensure that any effects, if obtained, were not due to participants misperceiving the auditory target words ${ }^{3}$. To assess the likelihood of misperceptions, we tested all auditory materials prior to the main experiments with an independent group of participants, using the gating procedure [11,35]. Via headphones (same equipment as in main experiments), participants heard increasingly longer fragments (so-called gates) of the auditory stimuli, in 50-ms steps. That is, for a particular word, the first presentation involved the initial $50 \mathrm{~ms}$ of the auditory stimulus, the second presentation the initial 100 $\mathrm{ms}$ of the auditory stimulus, etc., up to the final presentation which involved the whole auditory stimulus. After each gate, participants were asked to write down the word they thought the fragment was excised from. They were also asked to indicate the confidence of their lexical choice. In all, 60 participants were tested with the gating procedure, and each participant received one third of the auditory materials, yielding a total of 38,680 data points. Overall, accuracy of auditory word recognition was very good. By the final gate, the words from the semantic condition were correctly identified in $97.5 \%$ of cases, the words from the phonological condition in $96.7 \%$ of cases, and the words from the mediated condition in $96.5 \%$ of cases, with mean confidence ratings of $9.7,9.8$, and 9.7 on a 10-point scale (with '10' denoting the highest confidence score). In particular, there was not a single case in which a (fragment from a) phonologically related word was erroneously identified as the picture name it was paired with in

\footnotetext{
${ }^{3}$ For example, if a phonologically related target was perceived as the object name (e.g. if Sand was misperceived as Hand) or a mediated target was perceived as the category associate's name (e.g. if Mus was misperceived as $F u \beta$ ), the resulting effects would be a consequence of identity or semantic priming rather than a consequence of phonological or mediated priming.
}

the main experiment at any of the gates. Likewise, there was not a single case in which a (fragment from a) mediated word was erroneously identified as the category associate's name it was paired with in the main experiment at any of the gates. In fact, there were only 16 cases (restricted to gates 1 and 2), in which the onset of the mediated word was misperceived as the onset of the competitor name (less than $0.05 \%$ of all data points). Mean recognition points for the words used in the semantic, phonological, and mediated conditions were $408 \mathrm{~ms}$ (S.D. $29 \mathrm{~ms}$ ), $448 \mathrm{~ms}$ (S.D. $26 \mathrm{~ms}$ ), and $439 \mathrm{~ms}$ (S.D. $34 \mathrm{~ms}$ ), respectively ${ }^{4}$.

Each experiment tested only two of the three related conditions and their corresponding unrelated control conditions. In Experiment 1, the semantic condition and the phonological condition were used. Eight different versions of the experiment were created. In a first step, the 48 target words for the semantic conditions and the 48 target words for the phonological conditions were divided into two subsets containing 24 words each. Two parallel raw lists were then created in the following way. In one list, the words from the first subsets of the words for the semantic and phonological conditions were combined with related pictures, and the second subsets with unrelated pictures. In the other list, the reverse was the case. This way, each target word appeared only once in each list, but contributed, across lists, to both experimental conditions (related vs. unrelated). In half of the experimental trials, positive probes were paired with the auditory target word, and in the other half of the trials negative probes were paired with the auditory target word. Positive and negative probes were equally distributed across experimental conditions. In each of the two raw lists, each prime picture was also presented twice in pure naming trials, in which the articulation cue immediately followed the picture display. These trials served as fillers only and were included to ensure that participants would actively engage in preparing the naming response from the onset of the prime picture.

Each of the two raw lists was then divided into four experimental blocks such that in each block each prime picture appeared exactly once. Across subsets of prime pictures, the assignment of the four conditions to the four blocks was counterbalanced using the random Latin square technique. In each block, half of the trials were filler trials and half were experimental trials with each of the four experimental conditions appearing exactly six times. For each block, two different pseudo-randomized sequences were created with the general criteria that (a) experimental conditions of the same type did not appear in succession,

\footnotetext{
${ }^{4}$ The recognition point of a word for a particular participant is defined by the gate at which the participant had guessed the correct word with a confidence rating of either 9 or 10 , without changing his or her guess subsequently [35].
} 
(b) that no more than three experimental trials and three filler trials were presented in adjacent trials, and (c) that no more than five identical probe verification responses were required in succession. Each participant received all four blocks of one raw version. For half of the participants, the presentation sequence of these blocks was reversed. Repetitions of the same prime picture across blocks were separated by at least ten intervening trials.

\subsubsection{Procedure}

Participants were comfortably seated in a dimly lit sound-proofed booth. Participants performed a delayed picture naming task. They were instructed to prepare their responses while the prime picture was presented. On $50 \%$ of all trials, the prime picture was followed by a response cue ('!!!' displayed in green color), and participants were instructed to initiate the overt picture naming response as quickly as possible on appearance of this response cue (see upper panel of Fig. 1). These trials served as filler trials only and ensured that participants would indeed prepare the naming response as quickly as possible.

The remaining $50 \%$ of the trials were critical experimen- tal trials. On these experimental trials, the picture presentation was followed by the presentation of the auditory target word, and only then was the response cue for the picture naming response presented. After the picture naming response, a visual probe word was presented which either matched or did not match the auditory target word, and participants had to indicate this mis-/match by pressing one of two corresponding buttons (see lower panel of Fig. 1). Participants pressed the left button labeled yes, if auditory and visual word matched and the right button labeled no otherwise. Participants were asked to move as little as possible during the presentation of the fixation signal and the pictures.

Before the experiment started, participants studied written instructions. The delayed naming task was introduced as the participants' primary task which they were instructed to perform with maximal speed and accuracy. For the subsequent probe verification task, accuracy rather than speed was emphasized. Participants also studied a booklet containing all picture stimuli used in the experiment. In the booklet, a name was printed next to each picture. Participants were instructed to use these words only for naming

(a)

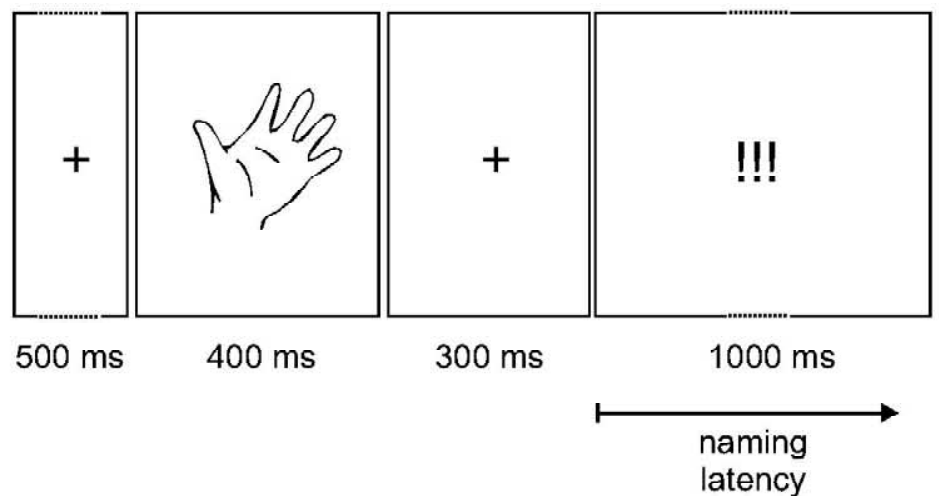

(b)

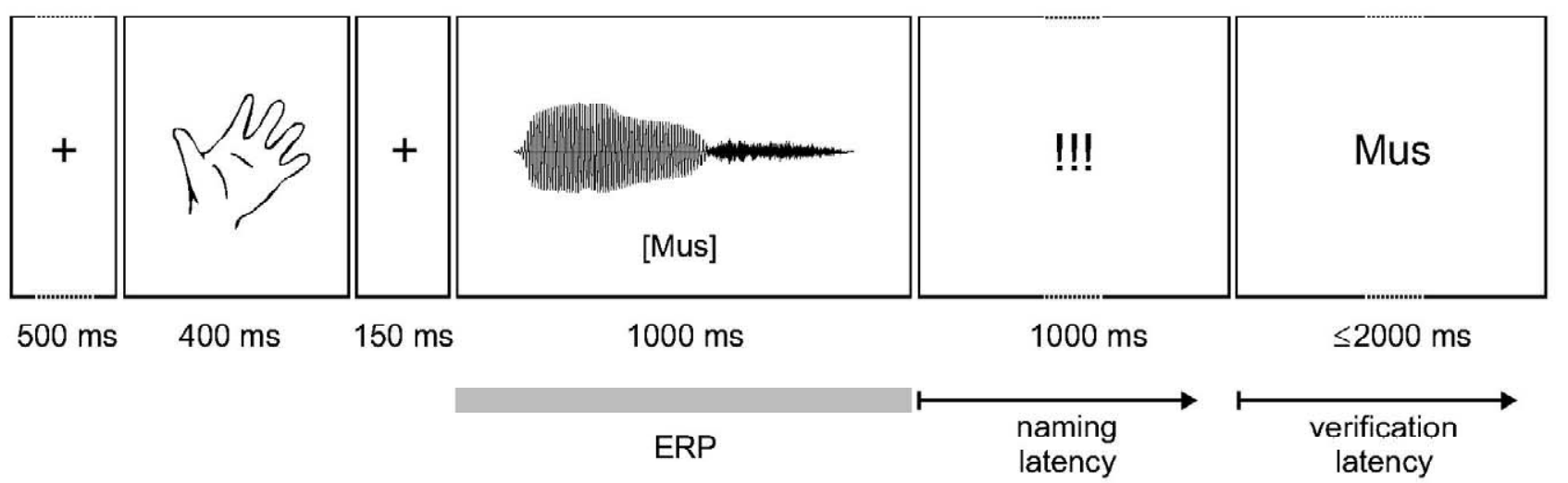

Fig. 1. Trial scheme for filler trials (panel a) and critical trials (panel b). 
the objects. Next, participants were trained on the delayed response task. In this phase, each experimental picture and each practice picture was presented once, yielding 57 practice trials. In a second step, auditory target words and visual probe words additionally appeared in half of the trials. During this training phase, only practice pictures and words were presented in another 36 practice trials. Then the main experiment started. After half of the trials, there was a short pause. Each of the two parts of the main experiment started with five warm-up trials containing practice pictures and words.

Prime pictures and probe words were presented centered on a CRT in light-gray on a black background. Probe words were presented in Helvetica typeface. Viewing distance was $\sim 100 \mathrm{~cm}$. The auditory target words were presented with headphones at a comfortable listening volume. Each trial started with a fixation signal ('+') presented for $500 \mathrm{~ms}$. It was replaced by the prime picture displayed for $400 \mathrm{~ms}$. At the offset of the prime picture, the fixation signal reappeared. In the filler trials, the fixation signal remained visible for $300 \mathrm{~ms}$, followed by the response cue presented for $1 \mathrm{~s}$. Then $2 \mathrm{~s}$ later, the next trial began. In the experimental trials, the fixation signal remained visible for $1150 \mathrm{~ms}$ after picture offset, and was then replaced by the response cue presented for $1 \mathrm{~s}$. The auditory target word started $150 \mathrm{~ms}$ after offset of the prime picture. The visual probe directly followed the response cue and remained on the screen until the participant pressed a button to indicate his/her probe verification response or, if no response was given, until $2 \mathrm{~s}$ had elapsed. After a further $2 \mathrm{~s}$, the next trial began. Naming latencies were measured from the onset of the response cue with a microphone feeding a voice-key connected to the computer. Observations were discarded and an error coded if (a) the picture was named other than expected, (b) no response was given within $1 \mathrm{~s}$, or (c) a response was initiated before the onset of the response cue or had a latency shorter than $50 \mathrm{~ms}$. Reaction times on trials in which the voice-key malfunctioned but the participant responded correctly were discarded, without coding an error.

\subsubsection{EEG recording}

The EEG was recorded monopolarly with $21 \mathrm{Ag} / \mathrm{AgCl}$ electrodes mounted in an elastic cap at the following positions: F7, F3, FZ, F4, F8, FC3, FC4, T7, C3, CZ, C4, T8, CP5, CP6, P7, P3, PZ, P4, P8, O1, and O2, referenced to the left mastoid. The horizontal electrooculogram $(\mathrm{EOGH})$ was recorded bipolarly from electrodes placed at the outer canthus of each eye, and the vertical electrooculogram (EOGV) was recorded bipolarly by electrodes placed above and below the right eye. Electrode impedance was kept below $5 \mathrm{k} \Omega$. Electrical activity was amplified within a band pass from d.c. to $40 \mathrm{~Hz}$. The EEG was digitized with a sampling rate of $250 \mathrm{~Hz}$. ERPs were computed from the onset of the target word for a duration of $1 \mathrm{~s}$ (i.e. to the onset of the response cue) relative to a 100-ms pre-stimulus baseline, separately for each of the four experimental conditions.

\subsubsection{Data analyses}

For each participant, mean amplitudes were computed for each electrode and each of the four experimental conditions, averaging over artifacts-free trials in which participants had given a correct response. Trials, on which a deviant naming response had been given, in which the response had been initiated before the onset of the response cue, with a latency shorter than $50 \mathrm{~ms}$, or with a latency exceeding $1 \mathrm{~s}$ were discarded from the analyses.

Analyses of variance were carried out on the semantic conditions (or mediated conditions, in Experiments 2-4) and the phonological conditions separately. For the analysis of the lateral electrodes, six regions of interest (ROIs) containing three electrodes each were defined: left-anterior (F7, F3, FC3), left-central (T7, C3, CP5), left-posterior (P7, P3, O1), right-anterior (F4, F8, FC4), right-central (C4, T8, CP6), and right-posterior (P4, P8, O2). The analysis of these electrodes included relatedness (related vs. unrelated), hemisphere (left vs. right), region (anterior vs. central vs. posterior) as completely crossed withinparticipant variables. The analysis of the midline electrodes included relatedness and electrode (FZ vs. CZ vs. PZ) as completely crossed within-participant variables. Where appropriate, the Greenhouse-Geisser correction for inhomogeneity of variance was applied. We report uncorrected degrees of freedom and probability levels following correction.

For each experiment, delayed naming latencies, probe verification latencies and the respective error rates in related and unrelated conditions were compared using $t$-tests. Latencies deviating more than 2 S.D.s from a participant's and an item's mean were discarded as outliers. We computed two analyses. In one analysis, participants were treated as random variable (participant analysis, $t_{1}$ ), and in a second analysis, items were treated as random variable (item analysis, $t_{2}$ ). When analyzing naming and size-judgment latencies and errors, the different prime pictures were considered as items, and when analyzing probe verification latencies and errors, the different probe words were considered as items.

\subsection{Results and discussion}

\subsubsection{Behavioral measures}

Table 1 displays reaction times and error rates for delayed naming and probe verification. There were no reliable differences between related and unrelated conditions (for delayed naming: all $P_{\mathrm{s}}>0.20$; for probe verification: all $P \mathrm{~s}>0.10$ ). In particular, there was no sign of a priming effect in the naming latencies. It has to be kept in mind, however, that the naming response in the present experiment was initiated on the basis of a cue, 
Table 1

Mean reaction times (in ms) and error rates (in \%) in delayed naming and probe verification from Experiment 1

\begin{tabular}{llllll}
\hline Condition & \multicolumn{2}{l}{ Delayed naming } & & \multicolumn{2}{l}{ Probe verification } \\
\cline { 2 - 3 } \cline { 6 - 6 } & Mean & $\%$ & & Mean & $\%$ \\
\hline sem-rel & $314(17)$ & $3.1(0.6)$ & & $566(27)$ & $1.6(0.5)$ \\
sem-unr & $317(15)$ & $3.1(0.8)$ & & $554(25)$ & $2.6(0.5)$ \\
pho-rel & $316(14)$ & $2.6(0.8)$ & & $559(25)$ & $1.3(0.4)$ \\
pho-unr & $314(15)$ & $1.7(0.5)$ & & $557(25)$ & $2.3(0.5)$ \\
filler & $369(15)$ & $1.5(0.3)$ & & $-^{\mathrm{a}}$ & $-{ }^{\mathrm{a}}$ \\
\hline
\end{tabular}

Standard errors in parentheses. filler, filler trials; pho-rel, target word phonologically related to the prime picture name; pho-unr, target word phonologically unrelated to the prime picture name; sem-rel, target word is the name of a semantic competitor of the prime picture name; sem-unr, target word is semantically unrelated to the prime picture name.

${ }^{a}$ No probes were presented in these trials.

which occurred $1350 \mathrm{~ms}$ after picture onset. This delay largely exceeds normal speeded picture naming latencies, and therefore lexical retrieval processes should have been fully completed by the time of cue onset. Presumably, the latencies actually measured just reflect the time needed for executing the prepared naming response. In line with this consideration, the naming latencies in the present experiment measured from response-cue onset were much shorter $(\sim 300 \mathrm{~ms})$ than regular speeded picture naming latencies measured from picture onset (usually $\sim 600 \mathrm{~ms}$ and above).

\subsubsection{ERP measures}

Based on the exclusion criteria, a total of $10.7 \%$ of all trials was discarded in Experiment 1, 8.1\% because of

\section{Semantic Conditions}
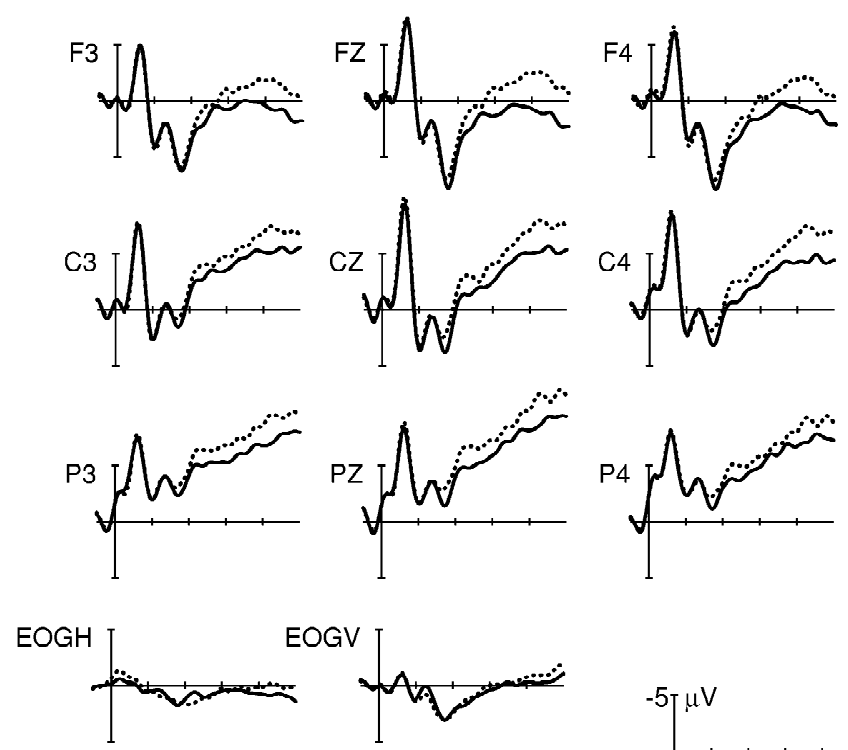

EEG artifacts, and $2.6 \%$ because of erroneous naming responses. The choice of time-windows selected for statistical analyses was based on a visual inspection of the grand average waveforms. At most electrode sites, semantic and phonological effects started at $\sim 400 \mathrm{~ms}$ after target onset and extended to the end of the recording epoch (1000 ms after target onset; Fig. 2 and top row of Fig. 6). As the effects obtained in Experiments 2-4 were also located in this time window, we decided to analyze this time window (400-1000 ms) in a consistent way, in all experiments and for all conditions.

2.2.2.1. Semantic conditions. In the analysis of the lateral electrodes, there was a significant main effect of relatedness $(F(1,31)=10.34, P<0.01, \mathrm{MSE}=12.72)$. The effect was broadly distributed, although not equally large across the different ROIs, as indicated by a significant interaction of relatedness, hemisphere, and region $(F(2,62)=6.48, P<$ $0.01, \mathrm{MSE}=0.20$ ). Subsequent analyses revealed that the condition effect was reliable in all ROIs except for the right-posterior one $(F(1,31)=3.71, P=0.06, \mathrm{MSE}=2.95)$. In the analysis of the midline electrodes, there was also a significant effect of relatedness $(F(1,31)=15.14, P<0.001$, MSE $=11.31$ ).

2.2.2.2. Phonological conditions. In the analysis of the lateral electrodes, there was a main effect of relatedness $(F(1,31)=17.51, P<0.001, \mathrm{MSE}=11.83)$. The effect was larger over the right hemisphere than over the left hemisphere $(F(1,31)=11.60, P<0.01$, MSE $=1.07)$, and in-

\section{Phonological Conditions}
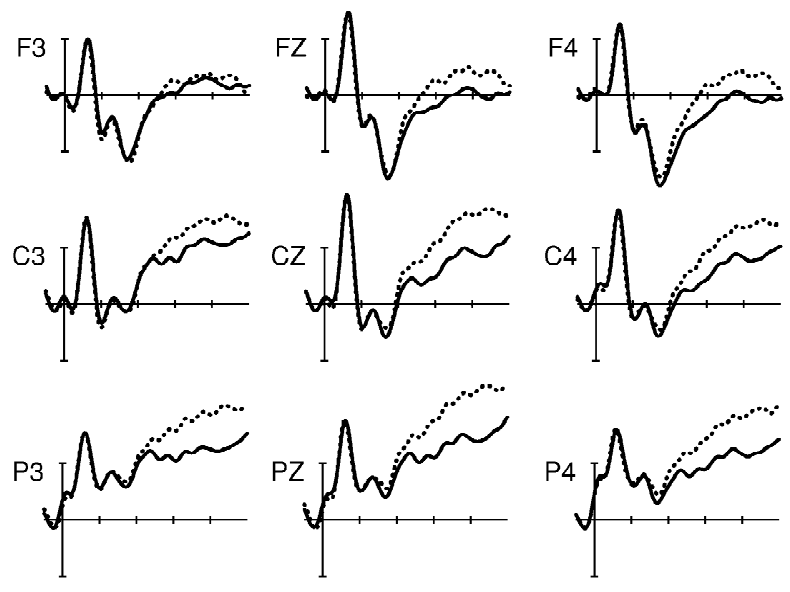

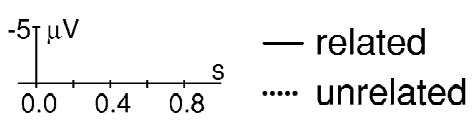

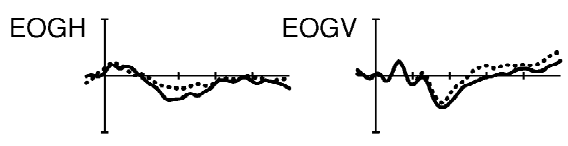

Fig. 2. Grand average ERPs $(n=32)$ for the semantic conditions (left panel) and the phonological conditions (right panel) at selected electrode sites, time-locked to the onset of the auditory target word from Experiment 1. Solid lines depict related conditions, and dashed lines depict unrelated conditions. In this and all following ERP figures, negative voltage is plotted up. For presentation purposes, the data were low-pass filtered with 10 Hz. All statistical analyses were performed on the unfiltered data. 
creased from anterior to posterior electrode sites $(F(2,62)=13.08, P<0.001, \mathrm{MSE}=1.26)$. There was also an interaction of relatedness, hemisphere, and region $(F(2,62)=3.51, \quad P<0.05, \quad \mathrm{MSE}=0.36)$, and subsequent analyses revealed that the effect of relatedness was significant at all but the left-anterior ROI. In the analysis of the midline electrodes, there was also a reliable effect of relatedness $\quad(F(1,31)=25.15, \quad P<0.001, \quad \mathrm{MSE}=11.52)$, which interacted with electrode site $(F(2,62)=5.49, P<$ $0.05, \mathrm{MSE}=1.63)$. Subsequent analyses revealed that a relatedness effect was obtained at all electrodes, but that it was larger at $\mathrm{CZ}$ and $\mathrm{PZ}$ than at FZ.

\subsubsection{Discussion}

If participants processed auditory target words while being engaged in preparing a picture naming response, the ERP to the words was less negative going if they denoted a category associate of the prepared picture name or were phonologically related to the prepared picture name. Both effects started $\sim 400 \mathrm{~ms}$ after target onset and exhibited a similarly broad distribution as in an earlier study using the same paradigm [17]. However, when compared to this study, the onset of the phonological effect was delayed, by some 100-150 ms. No comparable shift, however, was observed for the semantic effect. This difference in the timing of the phonological effect across studies is explained by differences in the materials used. In the previous study, primes and targets had shared initial segments, and in the present study, they had shared final segments. Hence, with the present materials, phonological priming could only become effective after the onset of the target word had been processed, thereby delaying its onset (for similar shifts in the onset of phonological N400 effects in auditory word-word priming studies, see Ref. [27] $)^{5}$.

In the present set of experiments, target words from the phonological condition shared the final segments with the picture name (e.g. Wand [wall], if the picture showed a hand), and target words in the mediated condition shared the final segments with the picture name's category associate (e.g. Mus [pap], if the category associate to the picture name is $F u \beta$ [foot]). Therefore, any potential effect in the mediated condition should be observed in the same time window as the effect in the phonological condition. For this reason, we used the same time window when analyzing these two conditions in the subsequent experiments that we had used in the present experiment (i.e. the time window 400-1000 ms).

Most importantly, the results from Experiment 1 demonstrate that semantic activation of category associates and

\footnotetext{
${ }^{5}$ The observed ERP effects do not show the exact signature of N400 priming effects as they are typically observed in, for example, wordword priming. The ERP effects in the present study start at $\sim 400 \mathrm{~ms}$ and extend to the end of the recording epoch. We will therefore refrain from referring to our ERP effects as N400 effects.
}

phonological activation of prepared picture names can reliably be observed with the selected materials. The next experiments, then, tested for the phonological activation of these category associates.

\section{Experiment 2}

Experiment 2 tested for the phonological activation of category associates, which had been shown to be semantically activated in Experiment 1, by replacing the target words denoting the category associates by phonologically similar words.

\subsection{Methods}

\subsubsection{Participants}

A total of 32 healthy participants (15 men and 17 women, with a mean age of 23.2 years, ranging from 20 to 29 years) were tested.

\subsubsection{Materials}

The materials were identical with those used in Experiment 1 with the only difference that the target words denoting the semantic competitors to the picture names were replaced by target words that were phonologically related to these competitors. Adjustments to the experimental lists were made where necessary to maintain the general randomization constraints.

\subsubsection{Procedure}

Same as in Experiment 1.

\subsubsection{EEG recording}

Same as in Experiment 1.

\subsubsection{Data analyses}

Same as in Experiment 1.

\subsection{Results and discussion}

\subsubsection{Behavioral measures}

Table 2 displays reaction times and error rates for delayed naming and probe verification. Delayed naming latencies were slightly faster in the phonological condition than in the unrelated control condition $\left(t_{1}(31)=3.05, P<\right.$ $\left.0.01 ; t_{2}(47)=3.11, P<0.01\right)$. None of the other effects was significant (for delayed naming: all $P \mathrm{~s}>0.10$; for probe verification: all $P \mathrm{~s}>0.30)$.

\subsubsection{ERP measures}

Based on the exclusion criteria, a total of $9.2 \%$ of all trials was discarded, $6.5 \%$ because of EEG artifacts, and $2.7 \%$ because of erroneous naming responses. Inspection of the waveforms for the mediated conditions revealed that ERPs to related and unrelated target words did not differ. 
Table 2

Mean reaction times (in ms) and error rates (in \%) in delayed naming and probe verification from Experiment 2

\begin{tabular}{llllll}
\hline Condition & \multicolumn{2}{l}{ Delayed naming } & & \multicolumn{2}{l}{ Probe verification } \\
\cline { 2 - 3 } \cline { 6 - 6 } & Mean & $\%$ & & Mean & $\%$ \\
\hline med-rel & $332(11)$ & $2.2(0.5)$ & & $604(39)$ & $2.7(0.9)$ \\
med-unr & $332(11)$ & $3.1(0.5)$ & & $601(36)$ & $2.2(0.6)$ \\
pho-rel & $325(11)$ & $3.5(0.7)$ & & $591(36)$ & $1.6(0.6)$ \\
pho-unr & $337(11)$ & $3.3(0.7)$ & & $590(35)$ & $2.2(0.5)$ \\
filler & $397(14)$ & $1.6(0.3)$ & & $-{ }^{\mathrm{a}}$ & $-{ }^{\mathrm{a}}$ \\
\hline
\end{tabular}

Standard errors in parentheses. filler, filler trials; med-rel, target word phonologically related to the category associate of the prime picture name; med-unr, target word phonologically unrelated to the category associate of the prime picture name; pho-rel, target word phonologically related to the prime picture name; pho-unr, target word phonologically unrelated to the prime picture name.

${ }^{a}$ No probes were presented in these trials.

By contrast, starting at $\sim 400 \mathrm{~ms}$ after target onset and extending to the end of the recording epoch, effects of phonological relatedness became visible (Fig. 3 and second row of Fig. 6). This latter finding further corroborates our decision of using a fixed time window across experiments (400-1000 ms), for the mediated conditions and the phonological conditions alike.

3.2.2.1. Mediated conditions. There was neither a main effect of relatedness $(F<1)$ nor any interaction of this variable with any of the topographic variables (for the two-way interactions: $F \mathbf{s}<1$, for the three-way interaction: $F(2,62)=2.16, P=0.13, \mathrm{MSE}=0.42)$ in the analysis of the lateral electrodes. There were no effects either in the analysis of the midline electrodes $\left(F_{\mathrm{s}}<1\right)$. To rule out the

\section{Mediated conditions}
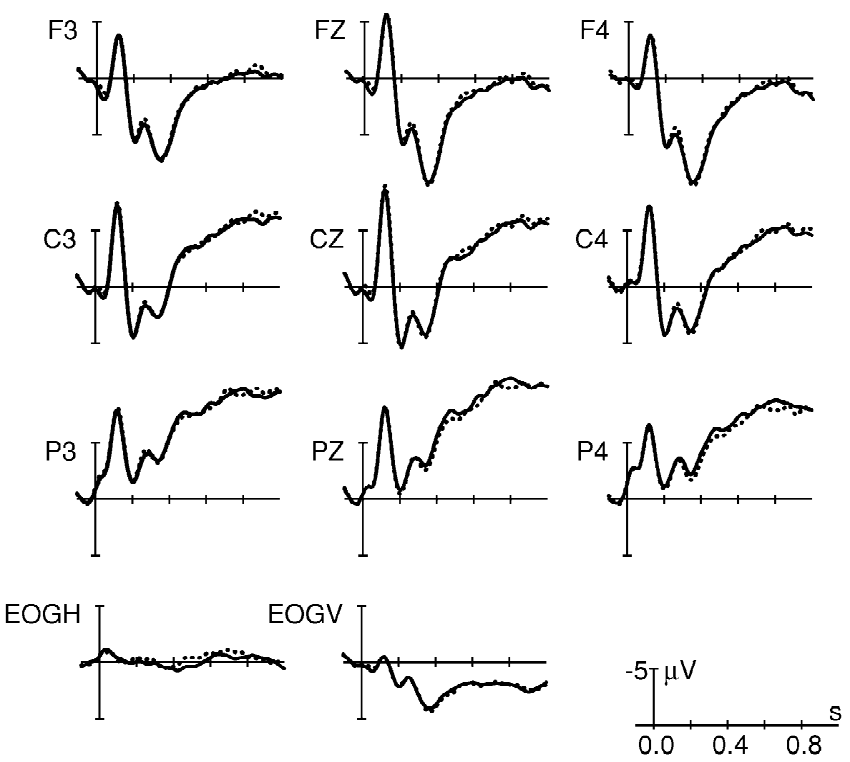

possibility that any small effect may have been missed by having used a time window which was too large, we performed additional analyses on successive $50-\mathrm{ms}$ time windows. These analyses revealed no significant main effect of relatedness in any time window tested. Occasionally, there was a significant interaction of this variable with (any of) the topographic variables, but subsequent analyses revealed that in all these cases the effects were due to non-significant trends that took different directions across topographic sub-regions.

3.2.2.2. Phonological conditions. ERP waveforms to phonologically related words were less negative going than ERP waveforms to unrelated words $(F(1,31)=13.16, P<$ $0.01, \mathrm{MSE}=15.04)$. The effect was larger over the right hemisphere than over the left hemisphere $(F(1,31)=9.38$, $P<0.01, \mathrm{MSE}=1.15$ ), and increased from anterior to posterior electrode sites $(F(2,62)=6.15, P<0.01, \mathrm{MSE}=$ 1.99). There was also a significant interaction of relatedness, hemisphere, and region $(F(2,62)=7.37, P<0.01$, MSE $=0.29$ ), and subsequent analyses revealed that the effect of relatedness was significant at all but the leftanterior ROI. The pattern was replicated in the analysis of the midline electrodes. There was a main effect of relatedness $(F(1,31)=10.89, P<0.01, \mathrm{MSE}=16.05)$ that interacted with electrode $(F(2,62)=3.28, P<0.05$, MSE $=1.73)$. Subsequent analyses showed that the effect of relatedness was significant at all electrodes but increased from anterior to posterior electrodes.

\subsubsection{Discussion}

The findings for the phonological conditions from

\section{Phonological conditions}
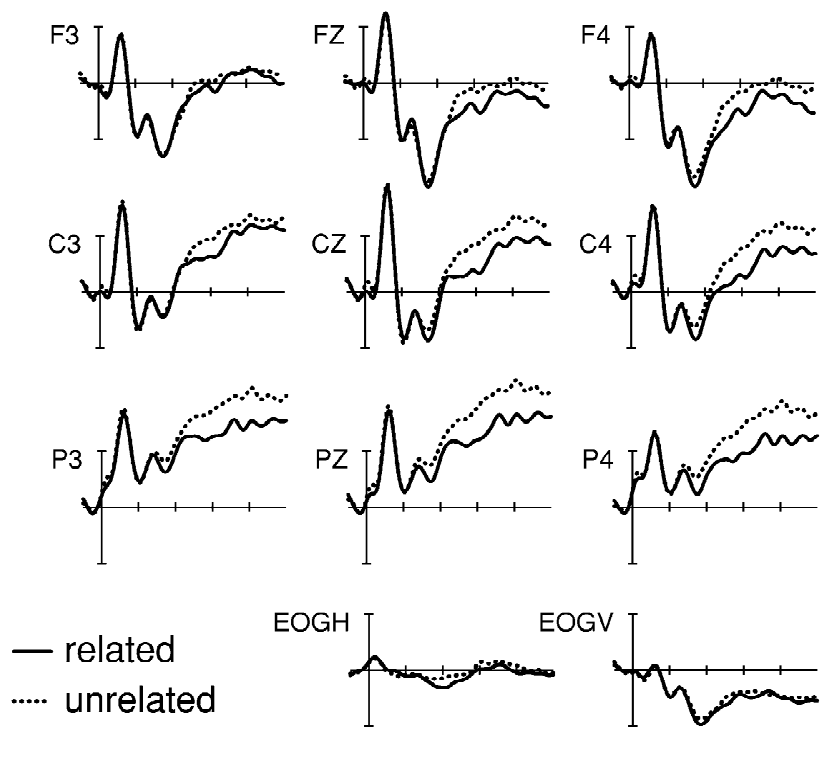

Fig. 3. Grand average ERPs $(n=32)$ for the mediated conditions (left panel) and the phonological conditions (right panel) at selected electrode sites, time-locked to the onset of the auditory target word from Experiment 2. Solid lines depict related conditions, and dashed lines depict unrelated conditions. 
Experiment 1 were fully replicated, demonstrating the sensitivity of the experiment. However, there was no trace of a mediated effect, suggesting that lexically activated semantic category associates (as established in Experiment 1) did not activate their phonological code to any measurable extent. However, before drawing strong theoretical conclusions based on a single null-effect, we wanted to test whether this observation is replicated in a situation in which the saliency of the mediated relation is enhanced.

\section{Experiment 3}

Experiment 3 was a replication of Experiment 2 with the only difference that the saliency of the mediated relation that held between a picture name and a target word was increased by having participants name the mediator (e.g. the picture of a foot by producing the word $F u \beta$ ) a few trials before the trial with the corresponding critical mediated condition (e.g. the word Mus [pap] following the picture Hand [hand]) was presented.

\subsection{Methods}

\subsubsection{Participants}

A total of 32 healthy participants (11 men and 21 women, with a mean age of 23.1 years, ranging from 18 to 30 years) were tested.

\subsubsection{Materials}

The basic materials were identical with those used in Experiment 2. However, in contrast to the previous experiments, pictures of the semantic competitors were also used. Each of these 48 additional pictures was presented twice in filler trials. These new trials were interspersed into the experimental lists such that one presentation of each picture preceded the corresponding critical (related or unrelated) mediated condition trial with a lag of three to six trials, while the second presentation was at a randomly chosen position in the experimental list. All of the four possible lags between induction trial and critical trials were used equally often. The same general randomization constraints as in Experiment 1 were applied, except for one difference. Due to the increased proportion of filler trials, up to five such trials could now follow in adjacent trials.

\subsubsection{Procedure}

Same as in Experiment 1.

\subsubsection{EEG recording. Same as in Experiment 1.}

\subsubsection{Data analyses. Same as in Experiment 1.}

\subsection{Results and discussion}

\subsubsection{Behavioral measures}

Table 3 displays reaction times and error rates for
Table 3

Mean reaction times (in ms) and error rates (in \%) in delayed naming and probe verification from Experiment 3

\begin{tabular}{llllll}
\hline \multirow{2}{*}{ Condition } & \multicolumn{2}{l}{ Delayed naming } & & \multicolumn{2}{l}{ Probe verification } \\
\cline { 2 - 3 } & Mean & & & Mean & $\%$ \\
\hline med-rel & $292(8)$ & $4.3(0.9)$ & & $574(22)$ & $3.1(0.6)$ \\
med-unr & $289(9)$ & $5.3(1.2)$ & & $556(22)$ & $1.8(0.6)$ \\
pho-rel & $294(7)$ & $3.4(0.9)$ & & $544(21)$ & $1.2(0.4)$ \\
pho-unr & $286(8)$ & $5.5(0.9)$ & & $560(22)$ & $1.4(0.4)$ \\
filler & $319(10)$ & $2.3(0.5)$ & & $-{ }^{\mathrm{a}}$ & $-{ }^{\mathrm{a}}$ \\
mediator & $344(11)$ & $4.1(0.6)$ & & ${ }^{\mathrm{a}}$ & $-{ }^{\mathrm{a}}$ \\
\hline
\end{tabular}

filler, filler trials; mediator, additional filler trials with picture of the semantic competitor; med-rel, target word phonologically related to the category associate of the prime picture name; med-unr, target word phonologically unrelated to the category associate of the prime picture name; pho-rel, target word phonologically related to the prime picture name; pho-unr, target word phonologically unrelated to the prime picture name.

${ }^{a}$ No probes were presented in these trials. Standard errors in parentheses.

delayed naming and probe verification. Delayed naming was slightly delayed in the related conditions, but the effects could not be confirmed in the item analysis (for the mediated conditions: $t_{1}(31)=2.38, P<0.05 ; t_{2}(47)=1.20$, $P=0.24$; for the phonological conditions: $t_{1}(31)=2.75$, $\left.P<0.05 ; t_{2}(47)=1.73, P=0.09\right)$. None of the other effects was significant (for delayed naming: all $P \mathrm{~s}>0.35$; for probe verification: all $P \mathrm{~s}>0.07)$.

\subsubsection{ERP measures}

Based on the exclusion criteria, a total of $14.7 \%$ of all trials were discarded, $10.1 \%$ because of EEG artifacts, and $4.6 \%$ because of erroneous naming responses. For the mediated conditions, visual inspection of the waveforms again did not reveal any systematic effect of relatedness. By contrast, the waveforms for the phonological conditions again diverged $\sim 400 \mathrm{~ms}$ after target onset at most electrodes and this effect extended to the end of the recording epoch (Fig. 4 and third row of Fig. 6).

4.2.2.1. Mediated conditions. In the analysis of the lateral electrodes, there was no main effect of relatedness and no interaction of this variable either with hemisphere or region (all $F_{\mathrm{S}}<1$ ). However, the three-way interaction of relatedness, hemisphere, and region was significant $(F(2,62)=3.81, P<0.05, \mathrm{MSE}=0.17)$. Subsequent analyses revealed that this interaction was due to a non-significant trend in the expected direction (less negative going waveforms in the related condition) at left posterior electrode sites $(F(1,31)=1.51, \quad P=0.23, \quad \mathrm{MSE}=2.12)$, while there was no such trend at any of the other ROIs (all $F \mathrm{~s}<1)$. The pattern was similar in the analysis of the midline electrodes. There was no effect of relatedness $(F<1)$, but a significant interaction with electrode site $(F(2,62)=3.42, P=0.05, \mathrm{MSE}=0.60)$. This interaction was due to a more negative going waveform in the related 
Mediated Conditions

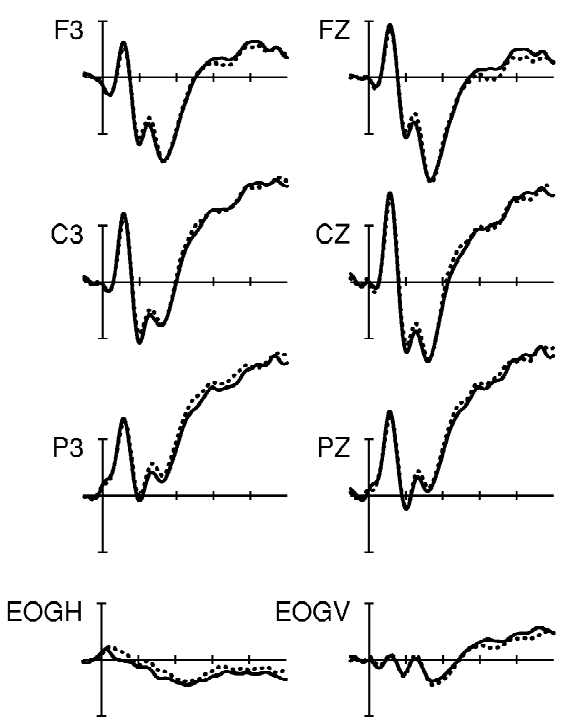

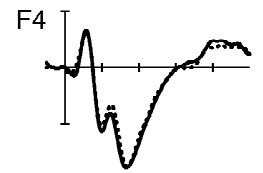
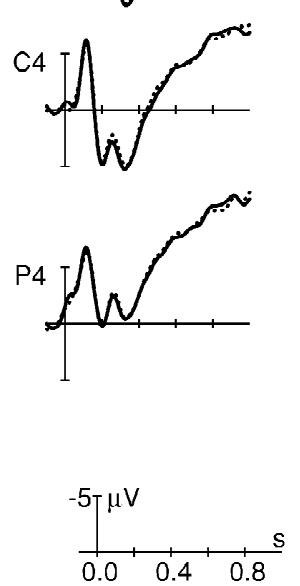

Phonological Conditions
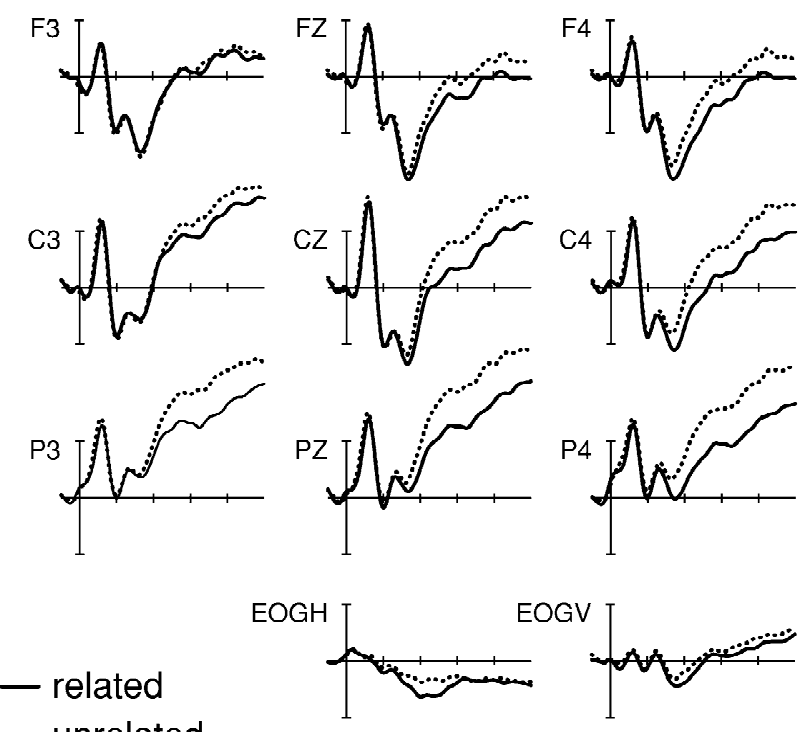

- related
..... unrelated

Fig. 4. Grand average ERPs $(n=32)$ for the mediated conditions (left panel) and the phonological conditions (right panel) at selected electrode sites, time-locked to the onset of the auditory target word from Experiment 3. Solid lines depict related conditions, and dashed lines depict unrelated conditions.

condition at FZ, but this unexpected trend was not reliable $(F(1,31)=1.19, P=0.28, \mathrm{MSE}=2.18)$. As in Experiment 2, additional analyses on successive 50-ms time windows were performed. In these analyses there was only a trend towards less negative going waveforms in the related condition in the time window 250-300 ms (for lateral electrodes: $F(1,31)=3.54, P=0.07, \mathrm{MSE}=9.07$; for midline electrodes: $F(1,31)=3.04, P=0.09$, MSE $=8.53)$. In the same time window, there was a marginally significant interaction of relatedness with the two topographic variables, and subsequent analyses revealed a significant effect of relatedness in the right anterior ROI $(F(1,31)=4.49$, $P<0.05, \mathrm{MSE}=3.42$ ), but not at any of the other ROIs $(P \mathrm{~s}>0.10)$. In other time windows, significant interactions of relatedness with (any of) the topographic variables were occasionally observed, but in none of these cases did further analyses identify a significant main effect of relatedness in any of the topographic sub-regions.

4.2.2.2. Phonological conditions. In the analysis of the lateral electrodes, there was a significant main effect of relatedness $(F(1,31)=24.06, P<0.001, \mathrm{MSE}=11.30)$. The effect was larger over the right hemisphere than over the left hemisphere $(F(1,31)=29.47, P<0.001, \mathrm{MSE}=1.35)$, and increased from frontal to posterior electrode sites $(F(2,62)=23.74, P<0.001, \mathrm{MSE}=1.01)$. The interaction of relatedness, hemisphere, and region was also significant $(F(2,62)=7.31, P<0.01, \mathrm{MSE}=0.22)$, and reflects the fact the significant effects of relatedness were obtained in all ROIs except for the left anterior one. In the analysis of the midline electrodes, there was a main effect of relatedness
$(F(1,31)=18.37, P<0.001, \mathrm{MSE}=14.43)$. This effect interacted with electrode site $(F(2,62)=11.43, P<0.001$, $\mathrm{MSE}=1.00$ ), reflecting an increase of the relatedness effect from anterior to posterior electrode sites.

\subsubsection{Discussion}

There was, again, a reliable phonological effect similar in timing and topography to those obtained in Experiments 1 and 2, but no evidence for a mediated effect. This was the case despite the fact that participants had named the category associate serving as the mediator just a few trials before the corresponding critical trial from the mediated condition was presented. The only potential exception to this pattern was a marginally significant effect for the mediated conditions in the time window 250-300 ms. However, this trend did not reach conventional levels of significance, did not replicate in any of the other experiments, and was obtained in an unexpected time window (in which no effect for the phonological conditions was found).

Before accepting the conclusion that there is no measurable mediated priming of semantic category associates during speech planning, however, one needs to consider the fact that Experiments 2 and 3 used different sets of target words in the phonological and in the mediated condition. Therefore, one possible reason for having repeatedly obtained phonological effects but having repeatedly failed to obtain mediated effects in the previous experiments could be differences in the susceptibility to priming across the target word sets used in the respective conditions. Experiment 4 tested this issue. 


\section{Experiment 4}

Experiment 4 used the same target words as Experiments 2 and 3, but the prime pictures were replaced by pictures of their category associates. This way, the target words that had failed to reveal mediated effects thus far now tested for phonological effects, and the target words that had successfully tapped phonological effects thus far now tested for mediated effects. To the extent that differences in the susceptibility to priming across target word set cannot be held responsible for the failure to obtain mediated effects, we expected to replicate the pattern from Experiments 2 and 3.

\subsection{Methods}

\subsubsection{Participants}

A total of 32 healthy participants (13 men and 19 women, with a mean age of 24.2 years, ranging from 18 to 29 years) were tested.

\subsubsection{Materials}

All prime pictures were replaced by pictures of their semantic competitors (e.g. the picture Hand [hand] was replaced by the picture $F u \beta$ [foot]), while the auditory target words were maintained. This way, the auditory target word that had occurred in the phonological condition (e.g. Sand [sand]) now figured in the mediated condition, and the auditory target word that had occurred in the mediated condition (e.g. Mus [pap]) now figured in the phonological condition. Two target words in the new mediated condition had to be replaced because they shared the consonantal onset with the new picture name. The general randomization constraints were identical to those used in Experiment 1.

\subsubsection{Procedure}

Same as in Experiment 1.

\subsubsection{EEG recording}

Same as in Experiment 1.

\subsubsection{Data analyses}

Same as in Experiment 1.

\subsection{Results and discussion}

\subsubsection{Behavioral measures}

Table 4 displays reaction times and error rates for delayed naming and probe verification. There were no reliable effects in the naming data (all $P \mathrm{~s}>0.50$ ). Probe verifications were slightly faster in the related conditions, but these effects could not be confirmed in the item analyses (for the mediated conditions: $t_{1}(31)=2.30, P<$ $0.05 ; t_{2}(94)=0.89, P=0.38$; for the phonological con-
Table 4

Mean reaction times (in ms) and error rates (in \%) in delayed naming and probe verification from Experiment 4

\begin{tabular}{llllll}
\hline Condition & \multicolumn{2}{l}{ Delayed naming } & & \multicolumn{2}{l}{ Probe verification } \\
\cline { 2 - 3 } \cline { 6 - 6 } & Mean & $\%$ & & Mean & $\%$ \\
\hline med-rel & $338(11)$ & $3.9(0.9)$ & & $546(17)$ & $2.1(0.8)$ \\
med-unr & $337(10)$ & $3.3(0.8)$ & & $559(18)$ & $2.6(0.6)$ \\
pho-rel & $338(11)$ & $4.0(0.8)$ & & $543(18)$ & $2.0(0.6)$ \\
pho-unr & $338(13)$ & $3.7(0.9)$ & & $558(20)$ & $2.1(0.5)$ \\
filler & $400(15)$ & $1.7(0.3)$ & & $-^{\mathrm{a}}$ & $-{ }^{\mathrm{a}}$ \\
\hline
\end{tabular}

Standard errors in parentheses. filler, filler trials; med-rel, target word phonologically related to the category associate of the prime picture name; med-unr, target word phonologically unrelated to the category associate of the prime picture name; pho-rel, target word phonologically related to the prime picture name; pho-unr, target word phonologically unrelated to the prime picture name.

${ }^{a}$ No probes were presented in these trials.

ditions: $\left.t_{1}(31)=2.13, \quad P<0.05 ; \quad t_{2}(94)=1.23, \quad P=0.22\right)$. None of the other effects was reliable (all $P \mathrm{~s}>0.50$ ).

\subsubsection{ERP measures}

Based on the exclusion criteria, a total of $14.7 \%$ of all trials were discarded, $11.2 \%$ because of EEG artifacts, and $3.5 \%$ because of erroneous naming responses. For the mediated conditions, visual inspection of the waveforms again did not reveal any systematic effect of relatedness. By contrast, the waveforms for the phonological conditions diverged $\sim 400 \mathrm{~ms}$ after target onset at most electrodes and this effect extended to the end of the recording epoch (Fig. 5 and bottom row of Fig. 6).

5.2.2.1. Mediated conditions. In the analysis of the lateral electrodes, there were no significant effects (all $F_{\mathrm{s}}<1$ ). In the analysis of the midline electrodes, there was also no effect of relatedness $(F<1)$ and no interaction with electrode site $(F(2,62)=2.09, P=0.15, \mathrm{MSE}=0.68)$. In the analyses on subsequent $50-\mathrm{ms}$ time windows, no significant effect of relatedness was obtained either. If a significant interaction of this variable with (any of) the topographic variables was occasionally observed, further analyses failed to identify a significant main effect of relatedness in any of the topographic sub-regions.

5.2.2.2. Phonological conditions. In the analysis of the lateral electrodes, there was a significant main effect of relatedness $(F(1,31)=4.05, P=0.05, \mathrm{MSE}=18.49)$. The effect was larger over the right hemisphere than over the left hemisphere $(F(1,31)=4.09, P=0.05, \mathrm{MSE}=1.46)$, and increased from frontal to posterior electrode sites $(F(2,62)=5.97, P<0.05, \mathrm{MSE}=1.81)$. The interaction of relatedness, hemisphere, and region was also significant $(F(2,62)=5.74, P<0.01, \mathrm{MSE}=0.33)$, and further analyses 
Mediated Conditions
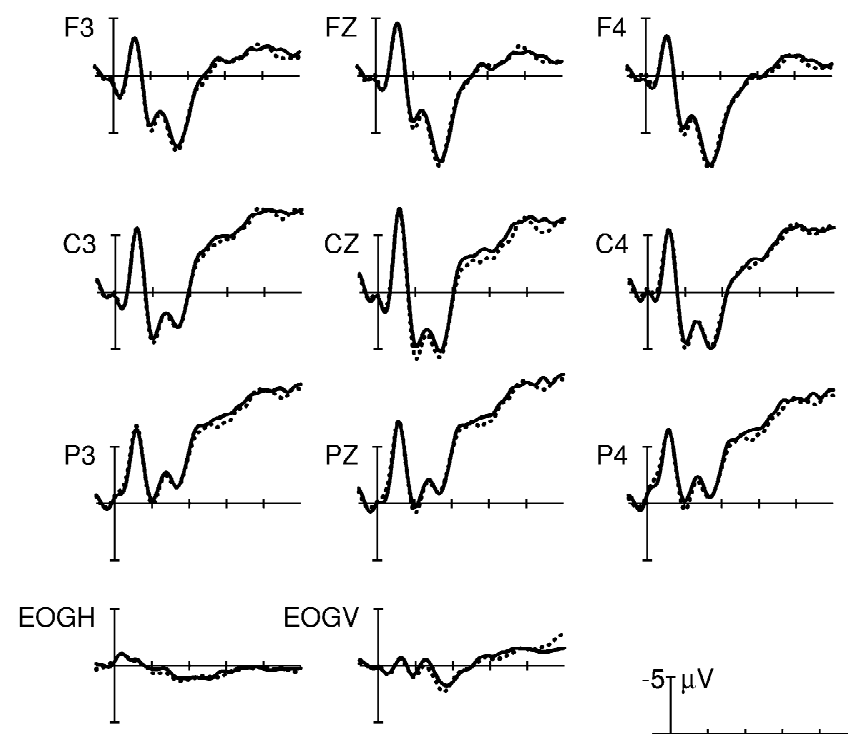

Phonological Conditions
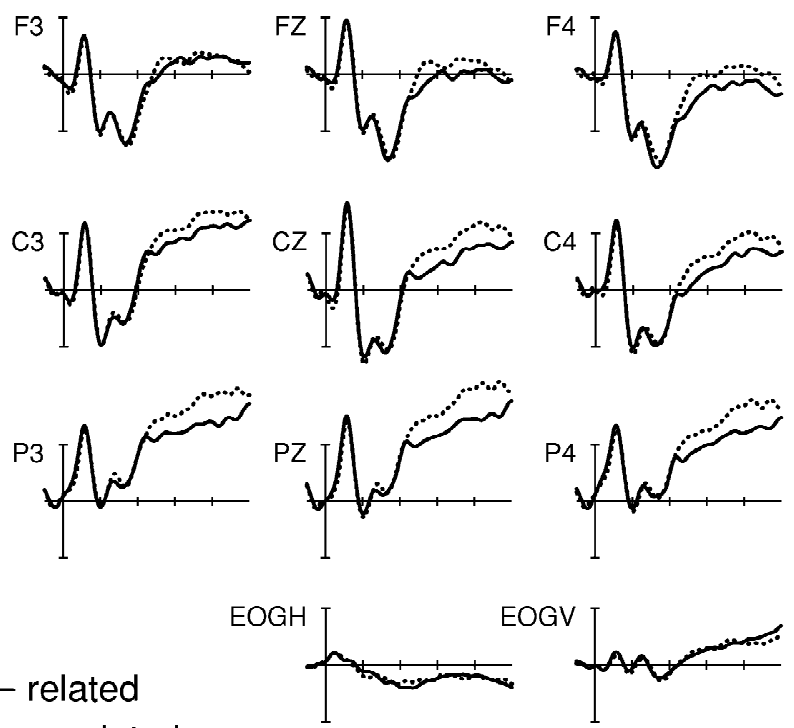

Fig. 5. Grand average ERPs $(n=32)$ for the mediated conditions (left panel) and the phonological conditions (right panel) at selected electrode sites, time-locked to the onset of the auditory target word from Experiment 4. Solid lines depict related conditions, and dashed lines depict unrelated conditions.

revealed that relatedness was only significant in the left and the right posterior ROIs and marginally significant in the right central ROI.

In the analysis of the midline electrodes, there was a main effect of relatedness $(F(1,31)=5.50, P<0.05$, MSE $=$ 17.75). This effect interacted with electrode site $(F(2,62)=$ 6.14, $P<0.01$, MSE=0.89), reflecting an increase of the relatedness effect from anterior to posterior electrode sites. Subsequent analyses revealed that the effect was significant only at $\mathrm{CZ}$ and PZ.

\subsubsection{Discussion}

When the target words that had thus far repeatedly failed to detect mediated priming effects were used to test for phonological priming effects, reliable effects were obtained. Although somewhat smaller, these effects had a similar topography and timing as the phonological effects observed in the previous experiments. Similarly, when the target words that had thus far reliably detected phonological priming effects were used to test for mediated priming effects, no effect was observed, as had been the case in the previous experiments. This pattern clearly rules out the possibility that incidental differences in the susceptibility to priming across target word sets can be held responsible for the failure of having detected mediated effects in the previous experiments. Rather, the results further corroborate our conclusion that semantically activated category associates do not activate their phonological code to any measurable degree.

\section{General discussion and conclusions}

In four experiments, lexical activation patterns during speech planning were explored using event-related brain potentials. Participants listened to target words while preparing a picture naming response. The ERP to the auditory target words was taken as an index of the lexical activation pattern induced by the picture-driven lexical retrieval procedure. Experiment 1 established that category associates had been semantically activated and that the picture name had been phonologically activated, as indexed by less negative going waveforms for target words that either denoted a semantic category associate of the prepared picture name (e.g. Fuß [foot], if Hand [hand] was the picture name, semantic effect), or that were phonologically related to the picture name (e.g. Sand [sand], phonological effect). These effects were widely distributed, started $\sim 400 \mathrm{~ms}$ after target onset and extended until the end of the recording epoch at $1000 \mathrm{~ms}$. Experiments 2-4 tested whether the semantically activated category associate was also phonologically activated (as predicted by non-discrete models of lexical retrieval) or not (as predicted by serial-discrete models). Experiment 2 replicated the phonological effect. However, there was no specific effect in the ERP to a target word that was phonologically related to the semantic category associate (e.g. Mus [pap], mediated semantic-phonological condition), suggesting that the category associate was not phonologically activated. Experiment 3 was a replication of Experiment 2 with one change in procedure. To enhance 


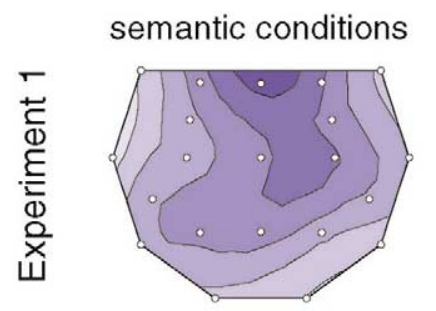

phonological conditions

mediated conditions

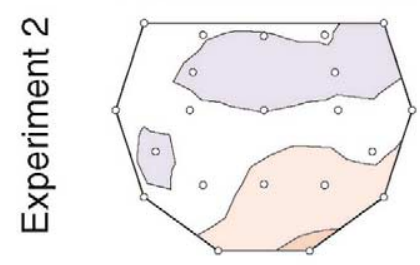

mediated conditions

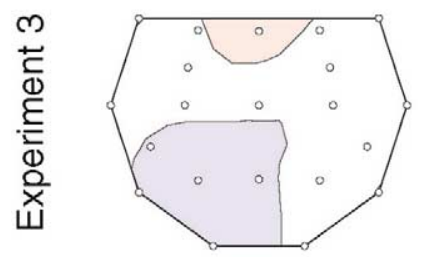

mediated conditions
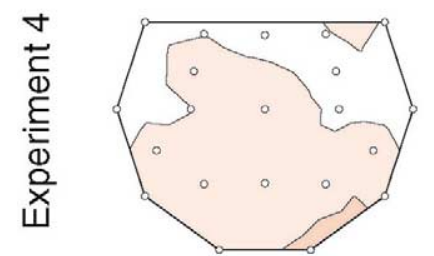

phonological conditions
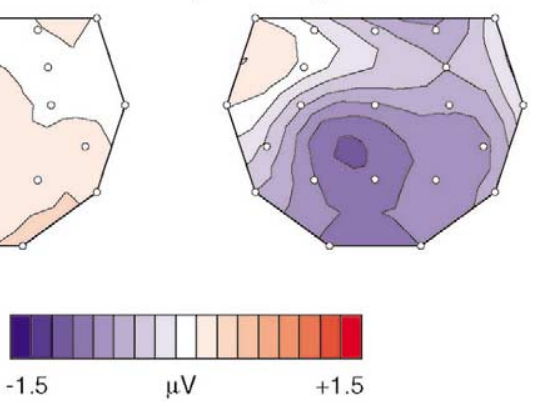

Fig. 6. Scalp distribution of the priming effects (unrelated-related) from Experiment 1 (top row; left panel: semantic conditions, right panel: phonological conditions) and Experiments 2-4 (second to fourth rows; left panel: mediated conditions, right panel: phonological conditions) for the time interval 400-1000 ms.

the saliency of the mediated relation that held between a picture name and a target word, participants named a picture of the category associate (e.g. $F u \beta$ [foot]) a few trials prior to the critical mediated trial. The results were similar to those from Experiment 2. There was, again, a phonological effect but no specific mediated effect. To rule out the possibility that the target word set used for tapping the mediated effect in Experiments 2 and 3 was not sensitive enough to reflect priming effects, the pictures in Experiment 4 were replaced by pictures of their category associates (e.g. Hand [hand] was replaced by $F u \beta$ [foot]). This way, the materials that had been used to tap mediated effects in Experiments 2 and 3 (e.g. Mus [pap]) now tested for phonological effects, and the materials that had been used to tap phonological effects (e.g. Sand [sand]) now tested for mediated effects. Clear phonological priming effects were once more obtained, while there was still no trace of a mediated effect, showing that material specific properties cannot be held responsible for the failure to detect mediated semantic-phonological effects in the previous experiments (see Fig. 6 for a summary of results for all four experiments).

As discussed in the introduction, such mediated effects, to the extent that they exist, must be assumed to be smaller, hence less easily detectable, than direct semantic or phonological effects. For this reason, we had hesitated to draw strong theoretical conclusions from the absence of such an effect in Experiment 2, and had opted for the strategy of cross-experiment replication, by systematically manipulating details of the experimental procedure in these replications (e.g. enhancing the saliency of the mediated relation or varying the target word set). The results were clear-cut. None of these experiments showed any indication of mediated priming, while always replicating the phonological priming effect. In a final attempt to detect mediated effects we performed a joint analysis on the mediated conditions from Experiments 2-4, enhancing the power of the statistical test by enlarging the participant sample by a factor of three ( $n=96$ participants). This analysis, once again, yielded unambiguous results. In the analysis of the lateral electrode sites, there was neither a significant main effect of relatedness nor a significant interaction with either hemisphere or relatedness $\left(F_{\mathrm{S}}<1\right)$. There was only a significant interaction of relatedness, hemisphere, and region $(F(2,62)=4.75, P<0.01, \mathrm{MSE}=$ $0.28)$, but subsequent analyses revealed that this effect was caused by a non-significant (and unexpected) trend towards a more negative waveform for the related condition at the right posterior ROI $(F(1,31)=2.34, P=0.13, \mathrm{MSE}=2.63)$, while no such trend was present at any of the other ROIs $(F \mathrm{~S}<1)$. In the analysis of the midline electrodes, there was no significant effect $(F \mathbf{s}<1)$. Analyses on consecutive 50-ms time windows also failed to obtain significant effects of relatedness in any time window. Thus, also this joint analysis failed to provide any evidence for mediated priming effects of category associates during speech planning. To put this pattern into perspective, it is important to recall that comparably subtle mediated effects have indeed been observed with electrophysiological measures in previous word recognition studies. For example, if participants performed a lexical decision to visually preceded words that were preceded by primes carrying a mediated relation to the target, the mediated prime-target relation manifested itself in a N400 effect but remained invisible in the simultaneously collected lexical decision reaction times [4]. This observation sharply contrasts with the findings from speech production studies. Neither extant behavioral reaction time studies [23,25], nor the present electrophysiological experiments found any comparable reflection of relations between a prepared picture name and 
a target word mediated by a semantic category associate. This consistent pattern suggests that, during speech planning, semantically activated category associates are not phonologically activated to any substantial degree.

The finding that semantically activated representations do not automatically activate their phonological codes is also in line with previous electrophysiological and behavioral findings. For example, it has been shown that a picture name's phonological code remains inactive if participants perform a non-lexical semantic task on the pictures [17]. Likewise, it has been demonstrated that a referent noun is semantically but not phonologically activated when speakers produce pronouns [20] (but see Ref. [31]; for further behavioral evidence see Refs. [2,23]). These findings clearly argue against models assuming unconditioned cascading of activation from a semantic representation level to a phonological representation level.

In summary, the present experiments show that category associates that are semantically activated during speech planning do not become phonologically activated to any measurable degree. No trace of such activation was obtained, even when using electrophysiological multi-dimensional measures with high time resolution, and when pooling data from a large number of participants across several experiments. This observation clearly sets strong constraints on the maximal amount of cascading that would be permissible in non-discrete models of lexical retrieval.

Notably, the now repeatedly demonstrated absence of phonological activation of category associates contrasts sharply with reports of corresponding effects for synonyms (like couch-sofa) $[18,25]$ and the more recent observation of such effects for super-/subordinate terms (like carpfish) [12]. An important question to be addressed in future research is whether this differential pattern is best explained as a consequence of particular representational features of the different semantic relations that hold between a target and its competitor in the framework of serial-discrete models, or as a consequence of optimum parameter setting in non-discrete models.

\section{Acknowledgements}

This work was supported by grant DFG Je229/4-1 and a Heisenberg fellowship from the German Research Council awarded to the first author. Thanks go to Kati Klische, Susanne Lamm, Kerstin Leukefeld, Frank Oppermann, Cornelia Schmidt, and Valentin Wagner for their assistance in data collection and analysis.

\section{References}

[1] S. Bentin, M. Kutas, S. Hillyard, Semantic processing and memory for attended and unattended words in dichotic listening: behavioral and electrophysiological evidence, J. Exp. Psychol. Hum. Percept. Perform. 21 (1993) 54-67.

[2] I. Bloem, W. LaHeij, Semantic facilitation and semantic interference in word translation: implications for models of lexical access (submitted for publication).

[3] A. Caramazza, How many levels of processing are there in lexical access?, Cogn. Neuropsychol. 14 (1997) 177-208.

[4] D.J. Chwilla, H.H.J. Kolk, G. Mulder, Mediated priming in the lexical decision task: evidence from event-related potentials and reaction time, J. Mem. Lang. 42 (2000) 314-341.

[5] M.F. Damian, R.C. Martin, Semantic and phonological codes interact in single word production, J. Exp. Psychol. Learn. Mem. Cogn. 25 (1999) 345-361.

[6] G.S. Dell, A spreading-activation model of retrieval in sentence production, Psychol. Rev. 93 (1986) 283-321.

[7] G.S. Dell, P.G. O'Seaghdha, Mediated and convergent lexical priming in language production: a comment on Levelt et al. (1991), Psychol. Rev. 98 (1991) 604-614.

[8] G.S. Dell, P.G. O'Seaghdha, Stages of lexical access in language production, Cognition 42 (1992) 287-314.

[9] G.S. Dell, M.F. Schwartz, N. Martin, E.M. Saffran, D.A. Gagnon, Lexical access in aphasic and non-aphasic speakers, Psychol. Rev. 104 (1997) 801-838.

[10] M.F. Garrett, Processes in language production, in: F.J. Newmeyer (Ed.), Language: Psychological and Biological Aspects, The Cambridge Survey of Linguistics, Vol. 3, Harvard University Press, Cambridge, MA, 1988, pp. 69-96.

[11] F. Grosjean, Gating, Lang. Cogn. Processes 11 (1996) 597-604.

[12] A. Hantsch, J.D. Jescheniak, H. Schriefers, Lexical activation patterns in semantic hierarchies during object naming (in preparation).

[13] T.A. Harley, Phonological activation of semantic competitors during lexical access in speech production, Lang. Cogn. Processes 8 (1993) 291-309.

[14] P.J. Holcomb, H.J. Neville, Semantic priming in visual and auditory lexical decision. A between modality comparison, Lang. Cogn. Processes 5 (1990) 281-312.

[15] P.J. Holcomb, J.E. Anderson, Cross-modal semantic priming: a time-course analysis using event-related brain potentials, Lang. Cogn. Processes 8 (1993) 379-411.

[16] J.D. Jescheniak, Word frequency effects in speech production, Doctoral dissertation, University of Nijmegen, Nijmegen, The Netherlands, 1994, 163 pp.

[17] J.D. Jescheniak, H. Schriefers, M.F. Garrett, A.D. Friederici, Exploring the activation of semantic and phonological codes during speech planning with event-related brain potentials, J. Cogn. Neurosci. 14 (2002) 951-964.

[18] J.D. Jescheniak, H. Schriefers, Serial discrete versus cascaded processing in lexical access in speech production: further evidence from the co-activation of near-synonyms, J. Exp. Psychol. Learn. Mem. Cogn. 24 (1998) 1256-1274.

[19] J.D. Jescheniak, H. Schriefers, Strictly discrete serial stages and contextual appropriateness, Behav. Brain Sci. 22 (1999) 47-48.

[20] J.D. Jescheniak, H. Schriefers, A. Hantsch, Semantic and phonological activation in noun and pronoun production, J. Exp. Psychol. Learn. Mem. Cogn. 27 (2001) 1058-1078.

[21] W.J.M. Levelt, Models of word production, Trends Cogn. Sci. 3 (1999) 223-232.

[22] W.J.M. Levelt, A. Roelofs, A.S. Meyer, A theory of lexical access in speech production, Behav. Brain Sci. 22 (1999) 1-75.

[23] W.J.M. Levelt, H. Schriefers, D. Vorberg, A.S. Meyer, T. Pechmann, J. Havinga, The time course of lexical access in speech production: a study of picture naming, Psychol. Rev. 98 (1991) 122-142.

[24] P.G. O'Seaghdha, J.W. Marin, Mediated semantic-phonological priming: calling distant relatives, J. Mem. Lang. 36 (1997) 226252.

[25] R.R. Peterson, P. Savoy, Lexical selection and phonological encod- 
ing during language production: evidence for cascaded processing, $\mathrm{J}$. Exp. Psychol. Learn. Mem. Cogn. 24 (1998) 539-557.

[26] P. Praamstra, D.F. Stegeman, Phonological effects on the auditory N400, Cogn. Brain Res. 1 (1993) 73-86.

[27] P. Praamstra, A.S. Meyer, W.J.M. Levelt, Neurophysiological manifestations of phonological processing: latency variation of a negative ERP component timelocked to phonological mismatch, J. Cogn. Neurosci. 6 (1994) 204-219.

[28] M. Radeaux, M. Besson, E. Fonteneau, S.L. Castro, Semantic, repetition and rime priming between spoken words: behavioral and electrophysiological evidence, Biol. Psychol. 48 (1998) 183-204.

[29] M.D. Rugg, Event-related potentials in phonological matching tasks, Brain Lang. 23 (1994) 225-240.

[30] M.D. Rugg, Event-related potentials and the phonological processing of words and non-words, Neuropsychologica 22 (1994) 435443.

[31] B. Schmitt, A.S. Meyer, W.J.M. Levelt, Lexical access in the production of pronouns, Cognition 69 (1999) 313-335.

[32] B. Schmitt, T.F. Münte, M. Kutas, Electrophysiological estimates of the time course of semantic and phonological encoding during implicit picture naming, Psychophysiology 37 (2000) 473-484.

[33] B. Schmitt, K. Schiltz, W. Zaake, M. Kutas, T.F. Münte, An electrophysiological analysis of the time course of conceptual and syntactic encoding during tacit picture naming, J. Cogn. Neurosci. 13 (2001) 510-522.

[34] H. Schriefers, A.S. Meyer, W.J.M. Levelt, Exploring the time course of lexical access in production: picture-word interference studies, J. Mem. Lang. 29 (1990) 86-102.

[35] P. Zwitserlood, The locus of the effect of sentential-semantic context in spoken-word processing, Cognition 32 (1990) 25-64.

[36] M. van Turennout, P. Hagoort, C.M. Brown, Electrophysiological evidence on the time course of semantic and phonological processes in speech production, J. Exp. Psychol. Learn. Mem. Cogn. 23 (1997) 787-806.

[37] M. van Turennout, P. Hagoort, C.M. Brown, Brain activity during speaking: from syntax to phonology in $40 \mathrm{~ms}$, Science 280 (1998) 572-574. 